\title{
Avant-propos au volume La notion de " chaîne opératoire " dans le monde : 50 ans d'études technologiques en Préhistoire
}

\author{
Yan Axel Gómez Coutouly \\ Maison Archéologie Ethnologie, UMR 7055, 21 allée de l’université, 92023 Nanterre cedex, France. \\ Email: yanaxel@northpacificprehistory.com
}

En 1964 s'est tenu aux Eyzies un symposium franco-américain auquel participèrent notamment Fr. Bordes, D. E. Crabtree et J. Tixier, décrit ensuite comme « une pierre de fondation de l'approche technologique » par C. Perlès en 1991. Un quart de siècle plus tard, faisant écho à cette rencontre, sont publiés les actes du colloque d'Antibes « 25 ans d'études technologiques en Préhistoire. Bilan et perspectives », confirmant la maturité de cette nouvelle démarche. Développée par l'école française (e.g., les travaux de P. Bodu, M.-L. Inizan, J. Pelegrin, C. Perlès, N. Pigeot, J. Tixier et B. Valentin entre autres), il s'agit d'une approche qui met en œuvre diverses notions, dont celle de chaîne opératoire (initialement introduite par M. Mauss et A. Leroi-Gourhan), de remontage mental, d'économie des matières premières et d'économie du débitage. Cette démarche analytique et systémique vise à isoler les variations ayant peu ou pas de valeur techno-culturelle telles que les accidents de taille, les pièces déviantes, le rôle du support, l'état technique de la pièce ou l'influence de la matière première. Un demi-siècle plus tard, où en est cette approche ? Le présent volume, en illustrant la pérennité de cette école de pensée, se veut un début de réponse. Au sein de ce numéro sont ainsi rassemblés divers cas d'étude où cette méthodologie est appliquée à différentes productions (sur galet, sur éclat, bifaciales, laminaires et lamellaires) provenant d'Europe, du Proche-Orient, d'Asie, d'Océanie et d'Amérique. En présentant les recherches d'auteurs issus d'équipes et de pays variés, l'ensemble met en évidence comment cette démarche a su s’intégrer aux problématiques de diverses équipes en France et à l'international.

L'article de F. Audouze et de C. Karlin est un historique reprenant toutes les étapes, les évolutions et les applications diverses aux travers des décennies de la notion de chaîne opératoire. Cette vision d'ensemble permet d'observer le long chemin parcouru par ce concept, illustré par de nombreux exemples. La suite du volume illustre comment cette notion a été appliquée à tout type de contextes et d'industries.

\section{Europe}

Ce continent est ici représenté par trois articles sur la France discutant du Paléolithique, du Mésolithique et du Néolithique. Tout d'abord, L. Klaric propose une étude des débitages lamellaires par la méthode du Raysse durant le Gravettien. L'auteur propose une analyse technologique fine, abordant de nombreux aspects comme les techniques, les gestes et le maintien dans ce type de débitage. À travers une analyse des productions laminaires et lamellaires au Mésolithique et au Néolithique, $\mathbf{P}$. Allard expose la variation des modalités de débitage mais également des techniques

Published by the School of History, Classics and Archaeology, University of Edinburgh ISSN: 2055-0472. URL: http://journals.ed.ac.uk/lithicstudies/

This work is licensed under a Creative Commons Attribution 2.5 UK: Scotland License. 
de débitage, dont la percussion indirecte et la pression, mettant en lumière une des approches largement développé par cette école technologique (la reconnaissance des techniques). L’étude de $\mathbf{M}$. Biard et C. Riche se centre sur la production d'éclats au Néolithique, des productions à priori expédientes et opportunistes. Grâce à une approche technologique et expérimentale, ils mettent en évidence des récurrences et concluent à une utilisation de percuteurs en silex pour ces productions.

\title{
Proche et Moyen Orient
}

Ces régions sont ici abordées à travers le prisme de la diffusion des débitages laminaires. D’un côté, F. Borell se centre sur l'origine et la diffusion des débitages laminaires bipolaires au Néolithique proche-oriental, lui permettant au passage d'aborder la question des spécialistes. De l'autre, J. Chabot étudie la diffusion des grandes lames au Néolithiques arménien, produites selon différentes techniques (percussion indirecte, pression debout et pression au levier).

\section{Asie}

Les articles de H. Forestier et al., deux grandes synthèses sur l'archipel indonésien et sur l'Asie du sud-est, montrent à quel point cette notion de chaîne opératoire peut être appliquée à des régions où le cadre chronologique est en pleine construction et où les études sur les industries lithiques ne sont pas toujours très développées. L’Extrême-Orient est ici représenté par une étude de $\mathbf{J}$. Pelegrin $\boldsymbol{e t} \boldsymbol{a l}$. sur une collection du Paléolithique supérieur du Japon, dont l'objectif est de développer un discours méthodologique sur la lecture technologique, notamment des techniques de taille, et sur les intentions de taille, ainsi que les implications de ces analyses. A. Hermann, grâce à ses travaux sur l'Océanie, nous fait ici découvrir des sociétés trop peu étudiées par le prisme de l'industrie lithique ; en effet, son analyse détaillée des lames d'herminettes montre le potentiel informatif de ce mobilier pour l'étude des sociétés et chefferies polynésiennes, abordant des sujets sociétaux comme la spécialisation.

\section{Amérique}

Au nord du continent, la région du Témiscouata au Québec (Canada) est une zone où les études technologiques sont rares. Ce vide est ici comblé par P. Eid avec une analyse technologique sur les industries des périodes Sylvicole et Contact, où l'analyse technologique est mise en parallèle avec la gestion des sources de cherts, permettant ainsi une réflexion sur l'économie de la matière première. Au sud du continent, A. Lourdeau offre une étude imbriquant le concept de chaîne opératoire et l'approche techno-fonctionnelle, appliquée aux productions du technocomplexe Itaparica du Brésil, lors de la transition Pléistocène-Holocène et durant l'Holocène ancien.

\section{Organisation éditoriale du volume}

Outre les auteurs, que je remercie pour leurs précieuses collaborations, je tiens également à faire part de ma gratitude auprès du comité scientifique spécialement mis en place pour ce volume pour leur aide (conseils, propositions d'auteurs, organisation du volume, sélection des relecteurs, etc.), ainsi qu’aux rapporteurs anonymes ayant accepté de relire les articles publiés dans ce volume.

Éditeur invité du volume en français :

Yan Axel Gómez Coutouly

UMR 7055 Préhistoire et Technologie (France)

Comité scientifique du volume :

Pierre Bodu

Adrian Burke

Otis Crandell

Ludovic Mevel

Jacques Pelegrin

Caroline M. Renard

\author{
CNRS, UMR 7041 ArScAn (France) \\ Université de Montréal (Canada) \\ Universidade Federal do Paraná (Brésil) \\ CNRS, UMR 7041 ArScAn (France) \\ CNRS, UMR 7055 Préhistoire et Technologie (France) \\ SDAVO ; UMR 7055 Préhistoire et Technologie (France)
}

